\title{
A aplicação experimental do Micro Learning na Educação Profissional e Tecnológica
}

Jaime Gabriel Mendonça de Oliveira, Universidade Federal de Santa Maria, jaime.oliveira@eb.mil.br, https://orcid.org/0000-0002-0630-5476

Mauro Leonardo Monteiro da Rosa, Escola Municipal de aprendizagem Industrial, monteiromauroster@gmail.com, https://orcid.org/ 0000-0003-0033-8397

Marcelo Freitas da Silva, Universidade Federal de Santa Maria, marcelo@ctism.ufsm.br, https://orcid.org/0000-0002-1476-5333 Leila Maria Araújo Santos, Universidade Federal de Santa Maria , leilamas@ctism.ufsm.br, https://orcid.org/0000-0002-1513-3717

Resumo: O presente artigo relata a aplicação do método Micro Learning (ML) na Educação Profissional e Tecnológica. Foi elaborado com base em um Estudo de Caso, realizado na Escola Municipal de Aprendizagem Industrial de Santa Maria. O ML é um método inovador, os dados coletados serviram de base para um mapeamento sobre o processo, o qual norteou todo o trabalho. O ML iniciou por meio de uma Avaliação Diagnóstica, a qual direcionou o planejamento e aplicação do ML.Por meio da proposta de ML, pretende-se auxiliar na comprovação de que o ML pode ser utilizado para novos ensinos, e que o tempo de duração pode melhorar a retenção do ensino e a atenção dos discentes. A pandemia trouxe limitações, e também a possibilidade de verificar o ensino e a importância de materiais didáticos bem confeccionados, alertando para o excesso ou falta de informações. Este estudo procura ainda, elencar algumas habilidades cognitivas desenvolvidas por meio do ML. Por fim, os dados apontados podem auxiliar no planejamento e aperfeiçoamento do método, aumentando sua utilização em nosso País.

Palavras-chave: Micro Learning, torno, usinagem, educação.

\section{The Experimental Application of Micro Learning in Education Professional and Technological}

\begin{abstract}
This article reports the application of the Micro Learning (ML) method in Professional and Technological Education. It was elaborated based on a Case Study, carried out at the Municipal School of Industrial Learning of Santa Maria. The ML is an innovative method, the data collected served as the basis for a mapping of the process, which guided all the work. The ML started through a Diagnostic Assessment, which guided the planning and application of the ML. Through the ML proposal, it is intended to help prove that the ML can be used for new teachings, and that the duration it can improve teaching retention and student attention. The pandemic brought limitations, as well as the possibility of verifying teaching and the importance of well-prepared teaching materials, warning of excess or lack of information. This study also seeks to list some cognitive skills developed through ML. Finally, the data indicated can help in the planning and improvement of the method, increasing its use in our country.
\end{abstract}

Keywords: Micro Learning, lathe, machining, education. 


\title{
1. INTRODUÇÃO
}

Otimizar o tempo se tornou uma das principais preocupações no dia a dia. Essa celeridade influencia no ritmo de trabalho, no cotidiano e principalmente na forma de ensinar. Inseridos nesse contexto, surgiram as Metodologias Ativas (MA) para uma melhor aplicação do ensino no ambiente escolar. Nas palavras de Moran, "são estratégias de ensino centradas na participação efetiva dos estudantes na construção do processo de aprendizagem, de forma flexível, interligada, híbrida" (MORAN, 2017, p. 2).

As MA buscam e apontam alternativas para uma melhor assimilação das disciplinas, procurando desenvolver ainda mais a autonomia do aluno. A otimização do tempo está interligada com as MA assim como a forma de aprender, no qual por meio destas metodologias, o nível de atenção dos alunos ganha novo destaque e é melhor trabalhada e direcionada tanto pelo professor como pelo teor de suas disciplinas.

Aliado as MA, o Micro Learning pode ser uma possibilidade de ensino rápido preciso e conciso. Segundo Gabrielli, Kimani e Catarci (2006, p. 13), define-se Micro Learning ${ }^{l}$ como:

\begin{abstract}
Uma nova área de pesquisa [...]. Baseia-se na ideia de desenvolvimento de pequenos pedaços de conteúdo, de aprendizagem e no uso de tecnologias flexíveis permitindo que os alunos possam acessá-los mais facilmente, em condições e momentos específicos, por exemplo, durante os intervalos de tempo (entre atividades) ou enquanto estão se deslocando. (tradução nossa).
\end{abstract}

Conforme salientam os autores, o ensino pode ser fracionado em pequenos pedaços, ocasionando ao seu término a construção de um conhecimento novo, possibilitando aumentar assim, a captação do conteúdo por parte do aluno, podendo esse estudar ou acessar o material disponibilizado em qualquer dispositivo, desde que utilizem tecnologia móvel²(GABRIELLI; KIMANI; CATARCI, 2006).

Por ser tratar de uma área nova de pesquisa, ainda há alguns hiatos que permeiam esse método nos estabelecimentos de ensino brasileiros. Porém, o estudo realizado por Alves (2020, p. 78), da Universidade de São Paulo (USP), propõe uma conceituação sobre o tema:

O Micro Learning é uma alternativa educacional predominantemente digital, que se utiliza de conteúdos curtos e objetivos, orientados para tópicos específicos, que pode ser utilizado na educação (corporativa) de forma estratégica, para intervenções que demandem agilidade e de forma sistemática, no apoio e na retenção de conhecimento compartilhado por meio de métodos diversos ou tradicionais.

Diante desse contexto, podemos inferir que ML é um método de ensino que pode ser utilizado em qualquer área de conhecimento de forma rápida, objetiva, precisa e concisa. O conteúdo é fracionado visando à otimização do tempo, e a possível retenção do ensino, levando em conta a nossa capacidade de atenção e memorização. É utilizado preferencialmente com tecnologias móveis, perante as facilidades de acesso

1 Micro Learning is a new research area [...] It is based on the idea of developing small chunks of learning content and flexible Technologies that can enable learners to Access them more easily in specific moments and conditions of theday, for example during time breaks or whileonthe move.

2Segundo a UNESCO, tecnologias móveis estão em constante evolução, podem ser resumidas em dispositivos móveis, digitais, facilmente portáteis com acesso a internet e recursos multimídia, como o smartphones, tablets, notebooks, leitores de áudio portáteis e jogos.

V. $19 \mathrm{~N}^{\circ} 2$, Dezembro, 2021 RENOTE

DOI: https://doi.org/10.22456/1679-1916.121371 
aos dados. Para sua utilização, o planejamento e seu design são alguns pontos de relevância que impactam sua aplicabilidade.

Buchen e Hamelmann (2010), salientam 5 (cinco) princípios básicos que devem ser considerados no design do ML, sendo eles: formato, foco, autonomia, estrutura e capacidade de endereçamento dos conteúdos. O formato do ML é intrinsecamente ligado ao tamanho, ou seja, pequenos pedaços de conteúdo podem auxiliar na assimilação. No tocante ao foco, ser o mais sucinto possível, expressar-se de maneira clara e precisa, no intuito de aumentar o foco sobre o assunto.

A autonomia é uma das principais características a ser alcançada pelo discente, permeia todo o método. O ML deve estimular o discente a procurar as soluções aos problemas, angariando novos conhecimentos. A estrutura do método é a forma de como o ML é desenvolvido, como por exemplo: título, tópicos, principais autores e datas. O endereçamento é o local que o aluno irá encontrar o conteúdo, qual plataforma de ensino? É no ambiente virtual da Escola? Foi endereçado direto aos e-mails dos discentes?

Durante esse Estudo de Caso, esses princípios foram os norteadores para a confecção de um modelo experimental. Este estudo foi desenvolvido na Escola Municipal de Aprendizagem Industrial de Santa Maria ${ }^{3}$. A observação se deu em uma aula de torno mecânico, na qual a proposta foi relembrar principais partes componentes do torno e a utilização de seus principais acessórios, teve a duração de 45 (quarenta e cinco) minutos e contou com a participação de 07 (sete) alunos e 02 (dois) professores.

Usinar uma peça requer coordenação motora e principalmente habilidades cognitivas, em especial a atenção e a motivação. O torno mecânico é uma máquina de corte, que utiliza várias ferramentas e quem opera essa máquina nesse estudo é o discente, o qual iniciou seus estudos em março de 2021.

Por se tratar de um curso totalmente voltado à Educação Profissional e Tecnológica, aproveitou-se os dias em que o discente estava em contato com o torno para a aplicação do método. O advento da pandemia do COVID-19 trouxe impactos para a formação dos alunos. Anteriormente à pandemia, as aulas expositivas eram realizadas de maneira presencial na sede da Escola, de segunda-feira a sexta-feira, mas, porém, devido às restrições sanitárias, os alunos passaram a frequentar a Escola de segunda-feira a quarta-feira, deixando os dias da semana restante para as aulas expositivas, em ambiente virtual.

Nesse cenário, o método ML foi empregado na confecção de um parafuso tipo "prisioneiro" (Figura 1), que requer por parte do discente, conhecimentos adquiridos anteriormente, e principalmente o conteúdo absorvido no material didático disponibilizado e na aula prática demonstrada pelo professor.

3A Escola Municipal de Aprendizagem Industrial de Santa Maria (EMAI) caracteriza-se por ser uma escola profissionalizante de Ensino Fundamental, nas áreas de Eletrometalmecânica e Desenho Técnico. Atende a alunos com matrícula concomitante na rede pública, no turno diurno, proporcionando-lhes qualificação profissional no contra turno de trabalho de sua escola de origem. Também oferece, no turno da noite, Educação Profissional Inicial Integrada ao Ensino Fundamental - Anos Finais - na modalidade de EJA, com formação em Eletricidade, Mecânica e Metalurgia (SILVA, 2003).

V. $19 \mathrm{~N}^{\mathrm{o}} 2$, Dezembro, 2021 RENOTE

DOI: https://doi.org/10.22456/1679-1916.121371 


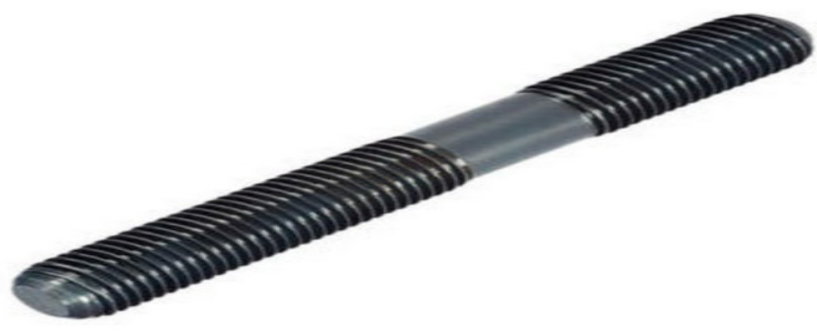

Figura 1 - Parafuso prisioneiro

\section{MATERIAL E MÉTODOS}

Inicialmente foi observado em sala de aula, o desenvolvimento da atividade. Essa etapa foi de suma importância para realização de um mapeamento do processo a ser estudado. Por meio desses foi possível constatar todo o percurso do material didático até o estado final da arte: a confecção da peça. Devido à pandemia, os professores disponibilizaram seus materiais didáticos no Google Classroom, para que seus discentes iniciassem seus estudos, anteriormente as aulas, sejam elas virtuais ou presenciais.

O material disponibilizado foi sobre as principais partes do torno mecânico ${ }^{4}$, bem como os principais acessórios. Juntamente com esse material, foram enviadas duas avaliações, para posterior envio de respostas, por meio do Ambiente Virtual de Aprendizagem.

Para trabalhar em um torno, é obrigatório saber e conhecer todas as suas partes e acessórios. Esse fato auxiliou na execução do ML, em especial na confecção do parafuso tipo "prisioneiro", pois é uma peça que consta do currículo da Escola e que requer do discente atenção, autonomia e prática. O método ML proposto é composto das seguintes fases: introdução, problema ou tarefa, resolução, discussão/reflexão e fechamento.

A introdução pode ser baseada em vídeos ou ser realizada de modo presencial. É o momento no qual o docente deve destacar a importância do assunto e sua aplicabilidade no cotidiano ou em oportunidades futuras. Sugere-se que a parte introdutória tenha no máximo de 02 (dois) minutos. A introdução ao assunto mais rápida é devido a otimização do tempo, para o restante do ML. É por meio da introdução que o aluno inicia seu processo de relembrar, realizando as suas sinapses ${ }^{5} \mathrm{e}$ assim acessando informações consolidadas.

Logo após, foi explanado sobre o problema ou tarefa e sobre a disponibilização de outras fontes de consulta. Devido à facilidade de acesso, o aparelho celular, surge como uma opção para consulta. Conforme Salinas e Marín (2014, p. 50):

É um uso muito comum entre a aprendizagem de adultos para resolver um problema ou erro. Nesses momentos, a mente fica mais receptiva a novas informações e mais disposta a aprender. Portanto, eles podem ser dispositivos

4Torno mecânico é a máquina-ferramenta, destinada a trabalhar uma peça animada de movimento de rotação, por meio de uma ferramenta de corte (FREIRE, 1984).

$5 \mathrm{~A}$ região de contato onde um neurônio transfere informação para outra célula. 
ideais para ajudar a obter informações como 'como fazer', tutoriais, manuais ou vídeos, ou busca de respostas 6 [...]. (tradução nossa)

Embora imersos em um ambiente de oficina e com as devidas precauções atinentes a segurança, foi permitido ao discente à consulta ao celular, ficando a cargo do mesmo a sua consulta, aqueles que optaram em não consultar outras fontes iniciaram à solução. Assim como na parte introdutória, o tempo sugerido é de 02 (dois) minutos.

A terceira fase é a resolução do problema ou tarefa, é a parte central do método ML. Nessa fase, é possível acompanhar toda a autonomia do aluno. Segundo Little (1991, p.4):

[...] a autonomia é uma capacidade - para desapego, reflexão crítica, tomada de decisão e ação independente. [...] implica, que o aluno irá desenvolver um tipo particular de relação psicológica com o processo e conteúdo de sua aprendizagem. [...] será exibida tanto na forma como o aluno aprende e na forma como ele ou ela transfere o que foi aprendido em contextos mais amplos. [...] implica que o aluno desfruta de um alto grau de liberdade ${ }^{7}$. (tradução nossa). (7)

Ainda nessa fase, é possível acompanhar o desenvolvimento de novas habilidades cognitivas, na qual a motivação para realização da tarefa deve ser mais aflorada. Segundo Barbosa (2005, p. 21), “A motivação é uma força que impulsiona o homem e é decisiva no desenvolvimento. Com a motivação há aprendizagem, pois, o ato de aprender é algo ativo e nunca passivo". É o momento também de verificar, se o material do didático está condizente com o problema. $\mathrm{O}$ discente tem aproximadamente 25 (vinte e cinco) minutos para a resolução.

Ao término da terceira fase e de posse da resposta, inicia-se a fase de discussão/ reflexão. Nessa fase, o aluno relata ao professor sobre as dificuldades ou facilidades encontradas perante a tarefa. O relato dos discentes é muito importante para a realimentação do método e do material didático disponibilizados.

A fase do fechamento é exclusiva do professor, é o momento de correção da avaliação. Perante os resultados, o docente pode analisar os índices de acertos. Sugerese que para uma comprovação efetiva do ML, os acertos devem estar em $80 \%$ e $90 \%$. Aos alunos que não atingiram esse nível de acerto é recomendável que refaçam todo o método ML, corrigindo os erros e assim reforçando o ensino.

Em 04 de outubro de 2021, na aula presencial, foi distribuído aos discentes um roteiro, o qual serviu de base para a realização do trabalho. Na parte expositiva, o docente demonstrou a toda a confecção do parafuso aos alunos e esses foram relembrados sobre as operações no torno mecânico e sobre o uso da ferramenta cossinete e tarraxa. Ao término da explanação, foram retiradas as dúvidas que surgiram e os alunos foram liberados para outras atividades na oficina.

No próximo dia, inicia-se o ML e o professor explica sobre a importância da peça e demonstra o uso do parafuso "prisioneiro" em outras peças. Os discentes de

6Es un uso muy común entre el aprendizaje de adultos con el objetivo de solucionar un problema o um error. Em esos momentos la mente es más receptiva a nueva información y tiene mayor voluntad de aprender. Por ello, pueden ser dispositivos ideales para ayudar a obtener información del tipo "como hacer", tutoriales, manuales o vídeos, o buscando las respuestas.

7Essentially, autonomyis a capacity - for detachment, critical reflection, decision making, and independentaction. It presupposes, bu talso entails, that the learner Will develop a particular kind of psychological relationt o the processand content of his learning. The capacity for autonomy Will be displayed both in the way the learner learn sand in the way He or she transfers what hás been learned to wider contexts. In common usa ge the word "autonomy" denotes a significant measure of independence from the control of others. The concept of learner autonomy similarly implies that the learner enjoys a high degreeof freedom.

V. $19 \mathrm{~N}^{0}$ 2, Dezembro, 2021 RENOTE

DOI: https://doi.org/10.22456/1679-1916.121371 
posse do roteiro são apresentados ao problema central da aula: confeccionar um parafuso "prisioneiro". Para a confecção da peça, foi estimado um tempo de 25 minutos, levando os alunos a uma situação de produção, em um ambiente fabril.

A primeira etapa da pesquisa foi a observação em sala de aula, a segunda foi a etapa foi a análise do conteúdo disponibilizado aos alunos. A terceira foi uma análise do roteiro junto com os professores, a quarta foi a execução do roteiro por parte dos docentes, no intuito de verificar o tempo e a complexidade.

O roteiro foi composto de 8 (oito) passos, são eles: preparo do material a ser utilizado, medição e chanframento das extremidades, seleção do cossinete de acordo com o diâmetro do material e o número de fios de rosca, seleção do porta cossinete, montagem da tarraxa no porta cossinete, prender o material nas castanhas do torno, início da rosca com o conjunto porta cossinete e tarraxa, sempre no sentido horário e com o número máximo de 2 (dois) fios de rosca, rosquear até a medida correta e retira a tarraxa no sentido anti-horário, respectivamente. Por fim, antes da execução foi confeccionado um mapeamento de todo o processo, o qual serviu de base durante toda a aplicação do método ML.

\section{RESULTADOS E DISCUSSÃO}

Os primeiros resultados obtidos de aplicação do método ML foram por meio de uma Avaliação Diagnóstica. Para fins de elaboração desse artigo, foi escolhido o questionário sobre os acessórios do torno mecânico, composto de 07 (sete) questões objetivas.

Esse questionário foi disponibilizado junto com o conteúdo didático, na correção anterior ao ML. Os 07 (sete) alunos do Curso de Usinagem Mecânica noturno, acertaram em média 6 (seis) questões cada um. Nas perguntas que compõem o questionário, o aluno encontrará todas as principais partes do torno bem como seus acessórios.

Quatro meses após a exposição ao conteúdo, aplicou-se o mesmo questionário no intuito de sondar e verificar os níveis de retenção. Os nomes dos discentes foram omitidos, deixando assim somente as iniciais dos nomes dos alunos. Os resultados obtidos constam na Tabela 1:

Tabela 1 - Mensuração de retenção

\begin{tabular}{l|l|c|c|c|c|c|c|c|c}
\hline \multirow{2}{*}{$\begin{array}{c}\text { Nr } \\
\text { Ordem }\end{array}$} & \multicolumn{1}{c|}{ Questão } & E.S. & A. S. & N. G. & S. D. & M. F. & W. O. & A. F. & TOTAL \\
\cline { 3 - 8 } 1 & $\begin{array}{l}\text { Qual o acessório que } \\
\text { permite fixar o material } \\
\text { com aperto simultâneo } \\
\text { das castanhas? }\end{array}$ & $X$ & $X$ & $X$ & $X$ & $X$ & $X$ & $X$ & $100 \%$ \\
\hline 2 & $\begin{array}{l}\text { Qual a parte do torno } \\
\text { que estão adaptadas as } \\
\text { placas universais? }\end{array}$ & --- & -- & --- & --- & -- & $X$ & --- & $15 \%$ \\
\hline 3 & $\begin{array}{l}\text { Para que serve o porta- } \\
\text { ferramentas? }\end{array}$ & $X$ & $X$ & $X$ & --- & --- & --- & $X$ & $57 \%$ \\
\hline
\end{tabular}




\begin{tabular}{l|l|c|c|c|c|c|c|c|c}
\hline 4 & $\begin{array}{l}\text { Para que serve a ponta } \\
\text { giratória? }\end{array}$ & --- & --- & --- & --- & --- & $\mathrm{X}$ & --- & $15 \%$ \\
\hline 5 & $\begin{array}{l}\text { Qual a parte do torno } \\
\text { que é montada a ponta } \\
\text { giratória? }\end{array}$ & $\mathrm{X}$ & $\mathrm{X}$ & $\mathrm{X}$ & $\mathrm{X}$ & --- & $\mathrm{X}$ & $\mathrm{X}$ & $85 \%$ \\
\hline 6 & $\begin{array}{l}\text { A que corresponde o } \\
\text { angulo de } 60^{\circ} \text { da ponta } \\
\text { giratória? }\end{array}$ & --- & --- & --- & --- & --- & $\mathrm{X}$ & --- & $15 \%$ \\
\hline 7 & $\begin{array}{l}\text { Qual a finalidade das } \\
\text { lunetas? }\end{array}$ & $\mathrm{X}$ & --- & $\mathrm{X}$ & $\mathrm{X}$ & $\mathrm{X}$ & $\mathrm{X}$ & $\mathrm{X}$ & $85 \%$ \\
\hline 8 & $\begin{array}{l}\text { Cite os principais tipos } \\
\text { de lunetas? }\end{array}$ & --- & --- & --- & --- & $\mathrm{X}$ & --- & --- & $15 \%$ \\
\hline
\end{tabular}

Fonte: Elaborado pelos autores (2021).

Para fins de cálculo de acerto ou erro, foi utilizada a média aritmética baseado no número de questões. A The Forgetting Curve estudada por Hermann Ebbinghaus (1885), nos demonstra que no instante que o novo conhecimento é apresentado, a retenção é de $100 \%$ (cem por cento). Nas próximas horas e no próximo dia, a retenção cai para $40 \%$ (quarenta por cento) e conforme o avanço dos dias, e se não houver revisão do conteúdo, os índices irão cair exponencialmente.

No Curso em estudo, passaram-se mais de 04 (quatro) meses, e conforme os estudos da curva de esquecimento, os índices de retenção deveriam estar abaixo dos $20 \%$ (vinte por cento). Porém, como demonstra a Tabela 1 , os índices ficaram acima de $37,5 \%$ (trinta e sete vírgula cinco por cento).

As novas medidas sanitárias, devido à pandemia, fizeram com que a Escola se adaptasse a uma nova forma de ensinar e praticar os conteúdos disponibilizados. A parte prática é uma das mais importantes para a formação do Mecânico de Usinagem e a cada semana os discentes, mesmo que de maneira indireta, têm uma revisão sobre o conteúdo.

Eles mantiveram contato com o torno mecânico para novas aprendizagens e confecção de outras peças, que constam do currículo escolar. O método empregado tem como estado final da arte a confecção do parafuso tipo "prisioneiro", por ser tratar de uma peça que requer medidas corretas, e de fácil execução. Os resultados obtidos constam na Tabela 2 :

Tabela 2 - Índices de tempo e acertos na confecção do parafuso

\begin{tabular}{|c|c|c|c|c|c|c|c|c|c|}
\hline \multirow{2}{*}{$\begin{array}{c}\mathrm{Nr} \\
\text { Ordem }\end{array}$} & \multirow{2}{*}{ Questão } & \multicolumn{7}{|c|}{ DISCENTES } & \multirow[b]{2}{*}{ TOTAL } \\
\hline & & E.S. & A. $\mathbf{S}$. & N. G. & S. D. & M. F. & W. O. & A. $F$. & \\
\hline 1 & Tempo gasto para & $35 ' 22 "$ & $21^{\prime} 15^{\prime \prime}$ & $25^{\prime} 22^{\prime \prime}$ & $28^{\prime} 02^{\prime \prime}$ & $32^{\prime} 30^{\prime \prime}$ & $30^{\prime} 09^{\prime \prime}$ & $34^{\prime} 40^{\prime \prime}$ & $29^{\prime} 20^{\prime \prime}$ \\
\hline
\end{tabular}

8 In the Field of psychology the term Forgetting Curve describe show the ability of the brain to retain information decreases in time.

V. $19 \mathrm{~N}^{\circ} 2$, Dezembro, 2021

RENOTE

DOI: https://doi.org/10.22456/1679-1916.121371 


\begin{tabular}{c|l|c|c|c|c|c|c|c|c}
\hline & confecção & & & & & & & & \\
\hline 2 & $\begin{array}{l}\text { Número total de } \\
\text { questões/ acertos }\end{array}$ & $10 / 9$ & $10 / 9$ & $10 / 10$ & $10 / 9$ & $10 / 8$ & $10 / 9$ & $10 / 9$ & $10 / 9$ \\
\hline 3 & $\begin{array}{l}\text { Índice de medidas } \\
\text { corretas da peça }\end{array}$ & $90 \%$ & $90 \%$ & $100 \%$ & $90 \%$ & $80 \%$ & $90 \%$ & $90 \%$ & $91 \%$ \\
\hline $\begin{array}{c}\text { MÉDIA DE ACERTO } \\
\text { GERAL }\end{array}$ \\
6
\end{tabular}

Fonte: Elaborado pelos autores (2021).

A média de acerto geral atingiu ao planejado para a execução da tarefa, bem como o índice de medidas da peça. Em se tratando de mecânica de usinagem, uma peça de medidas incorretas, é considerada "morta" e deve ser descartada, ou seja, sem aproveitamento. A média do tempo sugerido excedeu em 4 (quatro) minutos, porém deve se considerar que algumas variantes surgiram no decorrer do processo, como por exemplo: torno mecânico muito utilizado e com pequenas avarias, pois há no Estabelecimento de Ensino cursos diuturnos e noturnos, a ansiedade pelo início da tarefa e o nervosismo, por se tratar de uma avaliação.

\section{CONCLUSÕES}

Por se tratar de um método inovador e relativamente novo, em termo acadêmico, o método proposto transcorreu bem em todas as suas fases. A Teoria da Carga Cognitiva, estudada por Sweller (2003) apresenta as limitações de nosso sistema de memória, sejam elas a memória de trabalho ${ }^{9}$ e a memória de longa duração ${ }^{10}$.

A Teoria da Carga Cognitiva baseia-se na utilização dos esquemas armazenados na Memória de Longo Prazo para contornar as limitações da Memória de Trabalho. Presume-se que os esquemas permitem que muitos elementos sejam tratados como um único elemento na Memória de Trabalho e, como resultado, mais capacidade de Memória de Trabalho é liberada (SWELLER, 2003).

De posse do roteiro distribuído pelos professores no dia anterior a pesquisa, infere-se que esse serviu de esquema ${ }^{11}$ para a execução da tarefa. $O$ estudo sobre o roteiro ajudou aos discentes a não sobrecarregar sua memória de trabalho. Infere-se ainda que, os alunos conseguiram transferir seus conhecimentos anteriormente adquiridos para hora da confecção da peça, não ocorrendo assim uma sobrecarga no seu span de dígi$\operatorname{tos}^{12}$. No tocante ao método proposto um dos seus aspectos mais importantes e de maior reflexão é sobre a complexidade do assunto a ser desenvolvido. Para desenvolver assuntos muito complexos, infere-se que o ML possa ser utilizado apenas para desenvolver um tópico e não para explicar todo o assunto.

Em assuntos de menor complexidade, o aluno pode ter melhor desempenho, pois devido ao fracionamento, o conteúdo se torna mais "leve" e tende a angariar a atenção e

9É uma memória transitória, on-line, onde são armazenadas e processadas as informações necessárias ao desempenho de uma tarefa que requer consciência.

10Memórias de longo prazo são aquelas que você pode recordar dias, meses ou anos após terem sido originalmente armazenadas.

11Esquema é definido como um construto cognitivo que permite tratar múltiplos elementos de informação como se fosse um único elemento, categorizado de acordo com a maneira como será utilizado.

V. $19 \mathrm{~N}^{\circ} 2$, Dezembro, 2021

RENOTE

DOI: https://doi.org/10.22456/1679-1916.121371 
o foco dos discentes. Esse fato pode ser constatado por meio do roteiro distribuído pelos professores para a execução da tarefa, que se mostrou excelente fonte de consulta. Em momento algum os alunos necessitaram buscar outras fontes, inferindo-se que a partir da boa elaboração de roteiros, o discente consegue fundir a parte prática com a teórica.

Por meio do ML foi possível constatar um acréscimo na autonomia dos discentes, mesmo que esses estivessem sob a pressão e nervosismo de uma avaliação. Em um ambiente fabril e de produção, a cobrança por resultado é inerente a profissão.

A parte prática sobressai sobre a parte teórica do conteúdo, se comparar os índices de acerto das tabelas. Por meio da Avaliação Diagnóstica, esse fato ficou ainda mais nítido comparado com os acertos do questionário. A parte prática faz com que o discente mantenha acima da média de $20 \%$ (vinte por cento) seu nível de retenção, mesmo sofrendo ação da curva de esquecimento.

Por meio das observações, em sala de aula foi possível constatar que algumas vezes o aluno utiliza parte ou acessórios do torno, mas não sabe sua nomenclatura, ainda assim sabe qual sua utilização. Infere-se que para elevar o nível teórico, o material didático deve ser revisado, despertando ainda mais a atenção dos alunos, na tentativa de reforçar o ensino. Na tentativa de manter a atenção do discente, explana Khan (2013), "É importante que os professores introduzam 'mudanças' em vários momentos durante a aula 'para reiniciar o relógio da atenção'. As mudanças sugeridas por Khan estão presentes no método proposto, pois além de ser dinâmico, mantém a atenção do aluno constantemente. A mudança sugerida acompanha todo o processo de produção, ou seja, se molda um pedaço de ferro bruto em uma peça utilizável. Por meio do método proposto, infere-se que os alunos mantenham sua atenção seletiva e foco. Conforme Bear, Connors e Paradiso(2017, p. 720):

A atenção seletiva, ou simplesmente atenção, é a capacidade de focalizar
determinado aspecto dos sinais de entrada sensoriais. No sistema visual, a
atenção permite-nos a concentração em um objeto entre muitos outros do
campo visual. Interações entre distintas modalidades também podem ocorrer.

A falta de atenção pode ocasionar a "perda" da peça, ficando suas medidas comprometidas. Em Mecânica de Usinagem o principal fator são as medidas precisas e a qualidade da mesma. O método proposto do ML é bastante dinâmico, inferindo-se que durante a execução da tarefa, e com base nos resultados obtidos, a atenção dos discentes manteve-se constante. Uma limitação encontrada para a elaboração do artigo foi relacionado com a avaliação. Segundo Allain; Gruber; Wollinger (2020, pg.54):

Não há sinais de provas escritas nem de outros mecanismos que muitos já chamaram de "tradicionais", referindo-se a uma tradição exclusiva do contexto escolar.

É claro que, o parafuso tem medidas corretas, e essas fizeram parte da obtenção do quantitativo de erro e acerto, mas como avaliar a destreza de um mecânico de usinagem? Uma resposta possível é pelo grau de acabamento de suas peças, mas esse fato é mais visível do que escrito. Desse modo, a avaliação constou das medidas do parafuso e da expertise dos professores para correção. Os conceitos apresentados sobre o tema podem instigar outros docentes a adoção desse método de ensino, dentro de suas áreas de conhecimento. Para tal, sugere-se que as etapas citadas no item 2 -

12É o máximo de números escolhidos ao acaso que uma pessoa pode repetir após ouvir a lista ser lida. O span de dígitos normalmente é sete, mais ou menos dois.

V. $19 \mathrm{~N}^{\mathrm{0}} 2$, Dezembro, 2021

DOI: https://doi.org/10.22456/1679-1916.121371

RENOTE 
MATERIAIS E MÉTODOS sejam respeitados, para dar mais confiabilidade e visibilidade no método mapeado.

\section{REFERÊNCIAS}

ALVES, M. M. Microlearning: possibilidades e desafios na educação corporativa. Tese (Programa de Pós-Graduação em Tecnologia da Inteligência e Design Digital) Universidade de São Paulo, São Paulo, p. 78, 2020.

ALLAIN, O.; GRUBER, C.; WOLILINGER, P. R. O que avaliar em Educação Profissional? Princípios epistemológicos da formação de trabalhadores. Avaliação da Educação Profissional e Tecnológica: um campo em construção. Brasília. Instituto Nacional de Estudos e Pesquisas Educacionais Anísio Teixeira. 2020.

BEAR, M. F.; CONNORS, B. W.; PARADISO, M. A. Neurociências: desvendando o sistema nervoso. Porto Alegre: Artmed, 2017.

BARBOSA, D. F. Motivação no trabalho. Revista de Ciências Empresariais, v. 2, n. 1, p. 20-25, 2005.

BUCHEN, I., HAMELMANN, H. Micro learning: a strategy for on going professional development. eLearningPapers. 2010.

FLASHCARD. Hermann Ebbinghaus - a pioneerofmemoryresearch. Disponível em: $<$ http://www.flashcardlearner.com/articles/hermann-ebbinghaus-a-pioneer-of-memoryresearch/>. Acesso em 4 out. 2021

GABRIELLI, S.; KIMANI, S.; CATARCI, T.The design of micro learningexperiences: a research agenda. In: HUG, T.; LINDNER, M.; BRUCK, P. A. (Eds.). Micro learning: Emergingconcepts, practicesand Technologies after e-Learning. Proceedingsof Micro learningConference, 2005: Learning \&Working in New Media. Innsbruck, Austria: Innsbruck University Press, 2006. p. 44-53.

KHAN, S. Um mundo, uma escola: a educação reinventada. Rio de Janeiro: Intrínseca, 2013.

LITTLE, David. Autonomy: Definitions, Issues and Problems. Dublin: Authentik, 1991.

MORAN, J. Metodologias ativas e modelos híbridos na educação. In: YAEGASHI, S. F. R. et al. (Eds.). Novas Tecnologias Digitais: Reflexões sobre mediação, aprendizagem e desenvolvimento. Curitiba: CRV, 2017. p. 23-35.

SALINAS, J., MARÍN, V. I. (2014). Pasado, presente y futuro del micro learning como estrategia para el desarrollo profesional. Campus Virtuales, v. III, n. 2, p. 46-64. Disponível em:www.revistacampusvirtuales.es. Acesso em: 04 out. 2021.

SWELLER, J. Evolution of human cognitive architechture. In: The Psychology of Learning and Motivation, por B. Ross, vol. 43, pp. 215-266. San Diego: Academic Press., 2003. 